\title{
Percutaneous device closure of a ruptured sinus of valsalva presenting in acute heart failure
}

\author{
Jayawardena J B ${ }^{1}$, Rahuman M B F ${ }^{1}$, Francis G R ${ }^{1}$, Niraj $\mathbf{M}^{1}$, Kumara A H W' \\ Journal of the Ceylon College of Physicians, 2017, 48, 46-47
}

\begin{abstract}
Aneurysms of sinus of valsalva are rare cardiac abnormalities ${ }^{1}$. Following rupture it presents with acute heart failure. Definitive treatment is surgical or device closure ${ }^{2}$. A 25-year-old female presented with decompensated cardiac failure following a ruptured sinus of valsalva aneurysm (RSOVA). She underwent percutaneous device closure at the Cardiology Unit of the National Hospital of Sri Lanka (NHSL) and had an uneventful follow up for one year. This is the first successful closure of a RSOVA by a Sri Lankan team of cardiologists. The procedure is safe and feasible and can be performed with available facilities in selected patients.
\end{abstract}

Key words: device closure, ruptured sinus of valsalva, acute heart failure

\section{Introduction}

Percutaneous device closure of RSOVA is a recognized therapeutic procedure with successful outcomes. It is preferred over open heart surgery in selected patients ${ }^{2}$. In Sri Lanka standard therapy has been exclusively surgical closure.

\section{Case report}

A-25-year old unmarried female from Ragama developed acute shortness of breath and cough, accompanied by exertional dyspnoea and orthopnoea. She was treated for a respiratory tract infection in the private sector. She developed ankle swelling and epigastric pain. As her symptoms worsened she was

\footnotetext{
${ }^{1}$ Institute of Cardiology, National Hospital of Sri Lanka.

Corresponding author: Jayawardena JB

E-mail: jayj4262000@yahoo.co.uk.
}

https://orcid.org/0000-0001-9047-4178 admitted to a Teaching Hospital. A chest $X$ ray revealed bilateral pleural effusions. This prompted transfer to Welisara Chest Hospital. Auscultation revealed a murmur and an echocardiogram showed a RSOVA shunting into the RA. On admission to cardiology unit NHSL respiratory rate was $28 / \mathrm{min}$, pulse rate was 116 , JVP was elevated with leg odema and hepatic tenderness. Air entry was reduced with stony dullness at lung bases. Blood pressure was $114 / 60 \mathrm{mmHg}$ and she had a continuous murmur best heard at upper left sternal edge.

Transesophageal echo (TOE) confirmed a RSOVA with shunting from non-coronary cusp into RA (Figure 1).

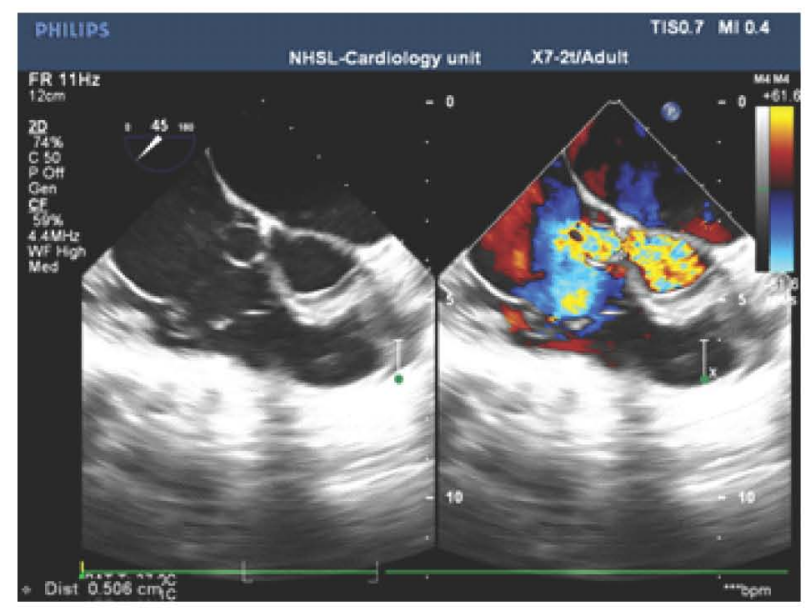

Figure 1. Demonstrates the ruptured sinus of valsalva shunting blood into the right atrium.

Treatment options were discussed with the patient and family. As open heart surgery with consequent precordial scar would bring an unfavourable social impact on a young female, device closure was preferred.

The procedure was performed using local anaesthesia under conscious sedation. Arterial and venous access was obtained via the right femoral artery and vein. $6 \mathrm{~F}$ and $8 \mathrm{~F}$ vascular sheaths were placed in the artery and vein respectively. Aortic pressure was $120 / 50 \mathrm{~mm}$ $\mathrm{Hg}$. Aortic angiogram showed a defect $7.66 \mathrm{~mm}$ in diameter at the neck. This was crossed from the aortic 
side using a $5 \mathrm{~F}$ Judkins right coronary catheter and a 0.035 " straight tip Terumo wire. A 0.035 " long guide wire was introduced and snared with a $10 \times 15 \mathrm{~mm}$ snare and exteriorized via the right femoral venous access. A 12/ 14 Coccoon PDA device was deployed across the defect using a 7F Cooks delivery sheath under TOE and fluoroscopic guidance after confirming the position of the aortic disk in the aortic cusp, the absence of valvular regurgitation, and the patency of the coronary vessels. Post procedure TOE and aortic angiogram revealed complete closure of the RSOVA without residual shunt (Figure 2).

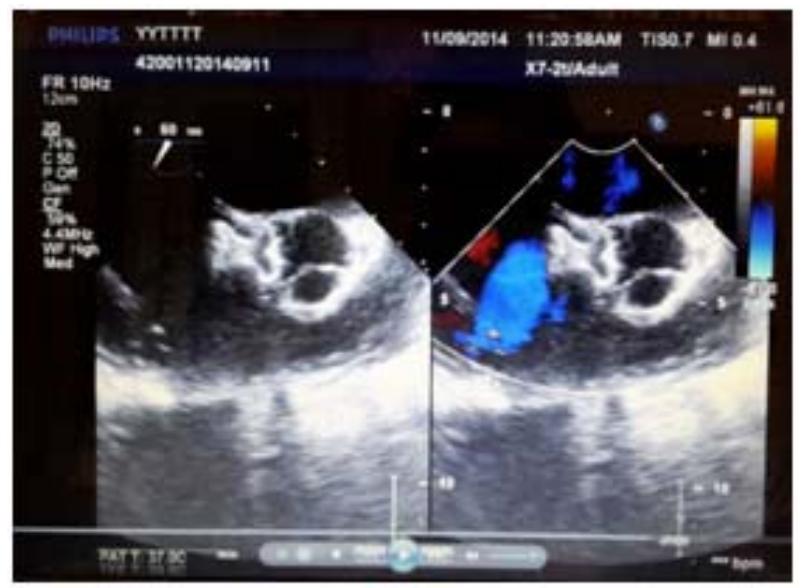

Figure 2. Demonstrates complete closure of the shunt by device.

Post procedure aortic pressures normalized to 135/ $80 \mathrm{~mm} \mathrm{Hg}$. Her symptoms resolved immediately and heart failure medications were stopped. She was discharged three days after the procedure on low dose aspirin for six months. The follow up period of one year was uneventful.

\section{Discussion}

Aneurysm of the sinus of valsalva is a thin walled tubular out pouching found usually in the right or noncoronary sinus of the aorta. It may be congenital or acquired. They are rare with a male-to-female ratio of $4: 1$ and a prevalence of $0.09 \%$ in a large autopsy series ${ }^{3}$. Commonest site for rupture is into the right atrium. This presented with features of acute cardiac failure. This is a very rare condition and can be easily missed unless suspected. Young age, acute heart failure and a continuous murmur are the clues for a correct diagnosis and echocardiography will confirm the diagnosis. Treatment options are surgical or device closure. Recent data has indicated a high degree of immediate to long-term success with percutaneous device closure 4 . Complications include device embolization, impingement on aortic valve causing aortic regurgitation, occlusion of coronary ostia, and infective endocarditis.

\section{Conflicts of interest}

There are no conflicts of interest.

\section{References}

1. Feldman DN, Roman MJ. Aneurysms of sinuses of Valsalva. Cardiology 2006; 106(2): 73-81.

2. Kuriakose EM, Bhatla P, McElhinney DB. Comparison of reported outcomes with percutaneous versus surgical closure of ruptured sinus of Valsalva aneurysm. Am J Cardiology 2015; 115(3): 392-8.

3. Prian GW, Diethrich EB. Sinus of Valsalva abnormalities. A specific differentiation between aneurysms of an aneurysms involving the sinuses of Valsalva. Vasc Surg. 1973; 7(3): 155-64.

4. Sinha SC, Sujatha V, Mahapatro AK. Percutaneous Transcatheter Closure of Ruptured Sinus of Valsalva Aneurysm: Immediate Result and Long-Term Follow-Up. Int J Angiol. 2015; 24(2): 99-104. 